\title{
Ysabel de Andia, La voie et le voyageur. Essai d'anthropologie de la vie spirituelle
}

Bibliographie, tableaux, index.

Paris, Éditions du Cerf, coll. « Théologies », 2012, 1024 p.

\section{Mustapha Naïmi}

\section{(2) OpenEdition}

\section{Journals}

Édition électronique

URL : http://journals.openedition.org/assr/26252

ISSN : $1777-5825$

Éditeur

Éditions de l'EHESS

\section{Édition imprimée}

Date de publication : 31 décembre 2014

Pagination : 106

ISBN : 978-2-7132-2467-6

ISSN : 0335-5985

\section{Référence électronique}

Mustapha Naïmi, «Ysabel de Andia, La voie et le voyageur. Essai d'anthropologie de la vie spirituelle », Archives de sciences sociales des religions [En ligne], 168 | 2014, mis en ligne le 27 mars 2015, consulté le 22 septembre 2020. URL : http://journals.openedition.org/assr/26252

Ce document a été généré automatiquement le 22 septembre 2020.

(c) Archives de sciences sociales des religions 


\section{Ysabel de Andia, La voie et le voyageur. Essai d'anthropologie de la vie spirituelle}

Bibliographie, tableaux, index.

Paris, Éditions du Cerf, coll. « Théologies », 2012, 1024 p.

Mustapha Naïmi

\section{RÉFÉRENCE}

Ysabel de Andia, La voie et le voyageur. Essai d'anthropologie de la vie spirituelle.

Bibliographie, tableaux, index. Paris, Éditions du Cerf, coll. « Théologies », 2012, 1024 p. 
1 Cet ouvrage est le fruit d'un cours sur les étapes dans la vie spirituelle à l'École cathédrale de Paris, au Collège des Bernardins; il présente la vie spirituelle comme une marche de l'homme en voyage. L'auteur considère que l'anthropologie chrétienne est théologique en ce sens que l'homme est vu dans la lumière, saint Irénée dirait dans la gloire, de Dieu qui se glorifie en lui. La raison de la création de l'homme n'est pas l'homme, mais la gloire de Dieu. Et cet acte divin de la création de l'homme est un acte souverain libre, gratuit, généreux ou amoureux.

2 L'ouvrage est un essai d'anthropologie spirituelle qui présente l'homme dans sa marche vers Dieu, de la terre au ciel. Il se structure d'une manière trinitaire : l'homme créé par Dieu, Père, Fils et Esprit
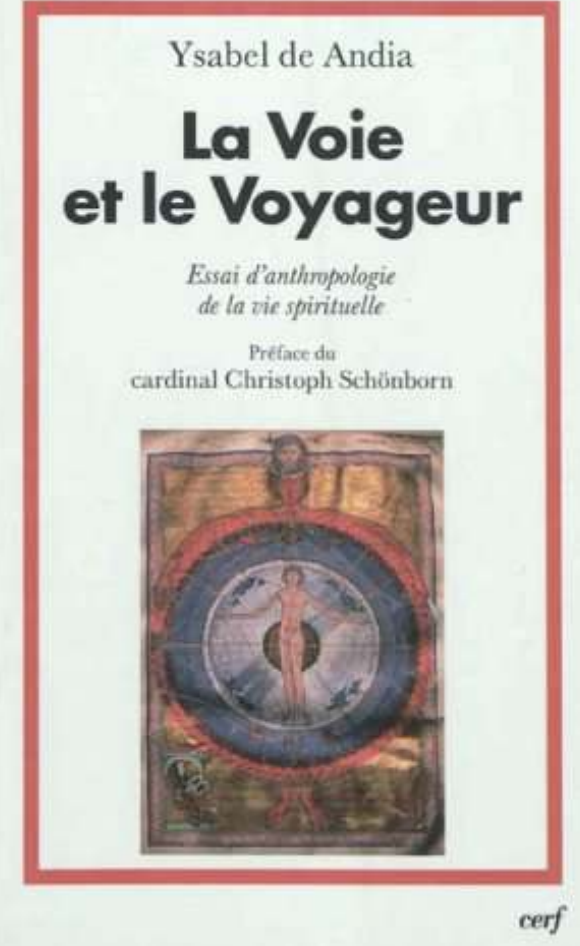
Saint, à son image et ressemblance, est racheté par le Christ, second Adam, et par l'Esprit Saint qui transforme l'homme tout entier, corps, âme et esprit. Ainsi l'homme est conduit à travers la mort à la vision de Dieu pour laquelle il a été fait et dans laquelle il trouve sa plénitude. Les thèmes retenus sont proprement spirituels : les larmes, la pureté du cœur, la virginité, etc. L'Esprit divinise l'homme tout entier : son regard, sa parole et son silence, son sommeil et ses veilles, c'est-à-dire ses yeux, sa bouche, ses lèvres, ses oreilles et son cœur. C'est dans la perspective eschatologique ouverte par la mort-résurrection du Christ que sont envisagés l'amour et la mort de l'homme, pour faire apparaître, à la fin des temps, la vision de l'homme nouveau.

3 Il ne s'agit pas d'écrire un traité d'anthropologie qui fixe l'homme en un instantané, mais de montrer cette progression de l'homme vers Dieu et cette croissance de l'homme en Dieu. L'image irénéenne d'Adam enfant manifeste ce désir de voir l'homme dans le dynamisme de sa propre croissance et de l'économie divine, parcourant les âges de l'homme qui sont aussi les âges de la vie spirituelle et parvenant à une ancienneté qui n'est pas celle du temps.

L'enjeu est donc l'unité de l'homme et le sens de sa vie. Ce n'est que dans le Christ que l'humanité trouve sa taille adulte et que l'homme vivant reflète la gloire de Dieu. Certes, cette anthropologie est un discours de la foi, théologique car fondé sur les vertus théologales, mais il signifie un investissement de la personne humaine et de son cheminement en faisant référence au domaine de l'esprit, de l'immatériel, de l'incorporel et de ce qui est relatif à l'âme. Le but de l'anthropologie spirituelle est de faire en sorte que les gens s'épanouissent le plus possible en actualisant leurs potentiels. Les apprentissages qu'elle propose reposent sur la psychologie et la théologie. Ils veulent faire redécouvrir l'importance des ressources spirituelles. La démarche proposée est d'entreprendre une réflexion sur sa manière de vivre ses 
relations à soi, aux autres et à Dieu ou à l'intelligence infinie, à la puissance éternelle et à bien d'autres selon ce que la personne conçoit de sa transcendance.

5 Ysabel de Andia livre là une synthèse de ses nombreuses études particulières sur les mystiques d'Orient et d'Occident, titre d'un ouvrage qu'elle a publié en 1994 dans la collection "Spiritualité orientale ». Elle traite d'une vision de l'homme profondément biblique, car c'est la Parole de Dieu qui donne, assure-t-elle, la lumière pour révéler l'homme à lui-même et les mots pour dire ce qu'il est dans la vision de Dieu. Il s'agit pour elle d'une "anthropologie spirituelle» qui a sa source dans la Bible présentée, selon l'expression monastique, comme une lectio divina enrichie par la méditation spirituelle des moines et des Pères, ou comme une auditio divina, car la source, c'est la Parole. Le texte de cette anthropologie est le texte inspiré de la Bible commentée par les traditions juive (pharisienne) et chrétienne qui, à la fois, récupère, comme les Hébreux, l'or des Égyptiens, la sagesse des philosophes grecs, leurs subtiles définitions et descriptions du voûç, de l'âme et de ses opérations, et exprime l'expérience spirituelle de générations de chrétiens. Il ne s'agit pas de partir d'une anthropologie philosophique, comme celle du De anima d'Aristote, ou des sciences humaines, mais de se placer dans une tradition spirituelle, celle du christianisme, qui livre, dans la variété des langues et des cultures, la richesse de ses approches et l'unité de sa conception de l'homme. Les termes mêmes de cette anthropologie sont bibliques et le vocabulaire grec s'enrichit des connotations hébraïques, comme le terme voûç, qui traduit souvent, dans la Septante, l'hébreu leb, le cœur, et s'enrichit ainsi de ses harmonies affectives, ou encore comme le terme latin simplex qui véhicule jusque dans la définition de Job comme homo simplex, par Grégoire le Grand, la richesse du terme hébreu tam, intègre et intégral, complet et simple, d'où parfait et juste. Il ne s'agit pas non plus d'écrire un « traité » d'anthropologie qui fixe l'homme en un « instantané », mais de montrer cette progression de l'homme vers Dieu et cette « croissance de l'homme » en Dieu.

6 Il s'agit en somme de l'unité et la divinisation de l'homme au terme desquels l'auteur fait deux remarques sur l'unité de l'homme et sa divinisation. Tout d'abord l'unité. Déjà au temps de l'hérésie de la Gnose, l'enjeu de la discussion entre les gnostiques et l'Église apostolique était l'unité de l'homme, et cette tentation gnostique s'est répétée de siècle en siècle jusqu'à nos jours. Si l'homme est principalement l'homme spirituel, et si l'homme charnel doit disparaître à la fin des temps, alors l'unité entre le charnel et le spirituel est détruite : la chair ne peut hériter du royaume des cieux, répètent à l'envi les gnostiques. Or, c'est la résurrection du Christ qui fonde l'unité même de l'homme, corps, âme et esprit, selon la doxologie de 1 Thessaloniciens $5: 23$, et la définition de l'homme spirituel est inséparable de la chair ressuscitée par l'Esprit. Inversement si l'on ne connaît pas dans l'homme comme les athées modernes, cette vie spirituelle ou de grâce, alors il n'y a pas dans l'homme de principe divin qui puisse le transformer; l'homme pense pouvoir se transformer lui-même par sa propre volonté, ce qui est un nouveau pélagianisme. Et non seulement l'existence de l'« esprit» (pneuma) est niée, mais également l'existence de "l'âme" (psyché) immortelle par une pensée qui est « matérialiste » sans se l'avouer, alors que les Grecs admettaient l'existence d'une âme immortelle.

7 Le second point est la divinisation de l'homme, rêve de l'humanité qui ne peut être atteint que dans le Christ. La tentation de l'homme a toujours été d'être comme Dieu, sans Dieu, de n'avoir d'autre origine ni d'autre loi que lui-même. "Dieu est mort » et l'homme n'a "rien » en face de lui-même: c'est l'athéisme nietzschéen. Or, Dieu a 
voulu, dans la création de l'homme et sa rédemption, partager sa gloire et sa nature pour que nous devenions " participants à la nature divine ». On dit en effet que Dieu et l'homme sont à la mesure de l'abaissement du Dieu-homme par " amour des hommes». Maxime dit encore que Dieu et l'homme se servent mutuellement de modèle, et que Dieu l'humanise pour l'homme, dans son amour de l'homme, dans la mesure même où l'homme, fortifié par la charité, se transpose pour Dieu en dieu. La divinisation de l'homme se fait par la philanthropie divine et la charité, à travers les mystères du Christ et la «suite du Christ ». Mais l'homme est également divinisé par l'esprit, qui en fait un homme spirituel vivant de la vie de l'Esprit. L'homme loin de Dieu avait des yeux et ne voyait pas, des oreilles et n'entendait pas et, de cette idole morte, l'Esprit a fait un homme vivant qui voit la Lumière et entend le Verbe divin. C'est enfin l'Esprit qui purifie le cœur de l'homme, cet homme intérieur que Dieu seul voit, pour en faire un homme selon le cœur de Dieu. 